\title{
SOBRE LA «ESPECIAL RESPONSABILIDAD»DEL TRIBUNAL DE JUSTICIA EN LA APLICACIÓN DE LA CARTA DE DERECHOS FUNDAMENTALES
}

\author{
PEDRO CRUZ VILLALÓN ${ }^{1}$ \\ Universidad Autónoma de Madrid \\ p.cruz@uam.es
}

Cómo citar/Citation

Cruz Villalón, P. (2020).

Sobre la «especial responsabilidad» del Tribunal de Justicia en

la aplicación de la Carta de Derechos Fundamentales.

Revista de Derecho Comunitario Europeo, 66, 363-384.

doi: https://doi.org/10.18042/cepc/rdce.66.02

\section{Resumen}

La garantía jurisdiccional de los derechos fundamentales en la UE ha hecho recaer en su Tribunal de Justicia una «especial» responsabilidad a lo largo del proceso de integración europeo. Esta particular responsabilidad se ha manifestado, sin embargo, de diferentes maneras a lo largo del tiempo. Durante el largo periodo precedente a la entrada en vigor de la Carta de Derechos Fundamentales esta responsabilidad se tradujo en la tarea de atraer al ordenamiento comunitario unos derechos fundamentales enraizados en un patrimonio europeo común. Desde que la Carta entrara en

\footnotetext{
Antiguo abogado general del Tribunal de Justicia de la UE. Presidente emérito del Tribunal Constitucional de España. Catedrático emérito de Derecho Constitucional de la Universidad Autónoma de Madrid.

El presente texto tiene su origen en la alocución con la que tuve el honor de intervenir en el I Seminario Gil Carlos Rodríguez Iglesias organizado por la Revista de Derecho Comunitario Europeo y celebrado en el Centro de Estudios Políticos y Constitucionales (Madrid) el 17 de febrero de 2020 bajo el lema «Diez años de aplicación judicial de la Carta de Derechos Fundamentales». He mantenido el lenguaje de mi intervención oral, renunciando a un aparato de notas en consonancia con las cuestiones esbozadas.
} 
vigor hace diez años, el Tribunal de Justicia se ha enfrentado con esa responsabilidad en un contexto enteramente diferente pero igualmente exigente para su función jurisdiccional.

\section{Palabras clave}

Derechos Fundamentales; Carta de Derechos Fundamentales de la Unión Europea; tradiciones constitucionales comunes; Convenio Europeo de Derechos Humanos; Tribunal de Justicia de la Unión Europea; valor jurídico de la Carta; tutela judicial.

\section{ON THE 'SPECIAL RESPONSIBILITY' OF THE COURT OF JUSTICE IN THE IMPLEMENTATION OF THE CHARTER OF FUNDAMENTAL RIGHTS}

\section{Abstract}

The jurisdictional guarantee of fundamental rights in the EU has placed on its Court of Justice a 'special' responsibility throughout the process of European integration. However, this particular responsibility has manifested itself in different ways over time. During the long period prior to the entry into force of the Charter of Fundamental Rights, this special responsibility resulted in the task of attracting fundamental rights to the Community legal system rooted in a common European heritage. Since the Charter entered into force ten years ago, the Court of Justice has faced that responsibility in an entirely different context but equally demanding for its jurisdictional role.

\section{Keywords}

Fundamental Rights; Charter of Fundamental Rights of the European Union; common constitutional traditions; European Convention on Human Rights; Court of Justice of the European Union; legal value of the Charter; judicial protection.

\section{SUR LA «RESPONSABILITÉ SPÉCIALE» DE LA COUR DE JUSTICE DANS LA MISE EN OEUVRE DE LA CHARTE DES DROITS FONDAMENTAUX}

\section{Résumé}

La garantie juridictionnelle des droits fondamentaux dans l'UE a conféré à sa Cour de justice une responsabilité «spéciale» tout au long du processus d'intégration européenne. Cependant, cette responsabilité particulière s'est manifestée de différentes manières au fil du temps. Au cours de la longue période précédant l'entrée en vigueur de la Charte des droits fondamentaux, cette responsabilité particulière 
s'est traduite par la tâche d'attirer les droits fondamentaux dans le système juridique communautaire, ancré dans un patrimoine européen commun. Depuis l'entrée en vigueur de la Charte il y a dix ans, la Cour de justice a fait face à cette responsabilité dans un contexte entièrement différent mais tout aussi exigeant pour son rôle juridictionnel.

\section{Mots clés}

Droits fondamentaux; Charte des droits fondamentaux de l'Union européenne; traditions constitutionnelles communes; Convention européenne des droits de l'homme; Cour de justice de l'Union européenne; valeur juridique de la Charte; protection judiciaire. 


\section{SUMARIO}

I. LA «ESPECIAL RESPONSABILIDAD»DEL TJ EN LA CONFIGURACIÓN DEL PARADIGMA JUDICIAL VIGENTE HASTA DICIEMBRE DE 2009. II. LA «ESPECIAL RESPONSABILIDAD» DEL TJ EN LA IMPLEMENTACIÓN DEL PARADIGMA CONSTITUCIONAL VIGENTE DESDE DICIEMBRE DE 2009: 1. El valor jurídico de la Carta. 2. El valor constitucional de la Carta. 3. La CDF y la cuestión de la pervivencia del antiguo paradigma.4. La CDF en el contexto del mandato de adhesión al CEDH. 5. Las indefiniciones del Título VII. 6. Las carencias de la tutela judicial de los derechos fundamentales. III. CONCLUSIÓN. BIBLIOGRAFíA.

«La protección de los derechos fundamentales en el sistema comunitario se caracteriza por dos factores, que vienen a determinar la especial responsabilidad que incumbe al Tribunal de Justicia en esta materia: de un lado, la inexistencia, en el ordenamiento jurídico comunitario, de un catálogo de derechos fundamentales de rango constitucional o legislativo; de otro, el carácter esencial del respeto de los derechos fundamentales en cuanto elemento básico del patrimonio común en el que descansan los propios fundamentos de la Comunidad y presupuesto indispensable para la adhesión de un Estado a ésta» ${ }^{2}$.

He vuelto a estas palabras con las que Gil Carlos Rodríguez Iglesias resumía magistralmente la problemática de la posición y la responsabilidad del Tribunal de Justicia de la Unión Europea (TJ, en adelante) respecto de la garantía de los derechos fundamentales en la UE en un tiempo ya pasado, en busca de inspiración en la tarea de situar y analizar esta misma problemática, ahora por referencia al singular periodo que constituyen los primeros diez años de aplicación judicial de la Carta de Derechos Fundamentales de la Unión Europea (en adelante, la Carta, o CDF).

\section{LA «ESPECIAL RESPONSABILIDAD» DEL TJ EN LA CONFIGURACIÓN DEL PARADIGMA JUDICIAL VIGENTE HASTA DICIEMBRE DE 2009}

Hace dos décadas, a la altura de 1998, Rodríguez Iglesias calificaba de especial la responsabilidad del TJ en lo referente a la garantía de los derechos

2 Gil Carlos Rodríguez Iglesias, Discurso de ingreso en el Academia Asturiana de Jurisprudencia, Oviedo, 1998. 
fundamentales en consideración a dos factores o circunstancias. La una no es privativa del TJ, contemplada en la perspectiva del espacio jurídico europeo. Se trata de la condición de los derechos fundamentales como «elemento básico del patrimonio común en el que descansan los propios fundamentos» de la hoy UE, así como «el carácter esencial del respeto de los mismos». Desde esa perspectiva, la posición de los derechos fundamentales no es muy distinta en la Unión de la que les corresponde en el ordenamiento constitucional de cada uno de los Estados miembros, como tampoco lo es la de los respectivos tribunales responsables de la eficacia de aquellos. Esta responsabilidad podía decirse especial para los tribunales tanto de la una como de los otros en razón del propio objeto de la respectiva actividad jurisdiccional, que no es otra que la eficacia del canon más elevado de toda comunidad política constitucionalmente organizada, en definitiva, la garantía de los derechos fundamentales. Con esto ya iba dicho también que los derechos y libertades no eran menos en la UE que en los Estados miembros, algo en lo que veinte años atrás no resultaba superfluo insistir.

Una particularidad en este punto cabría, sin embargo, deducir de la formulación elegida, la identificación de los derechos fundamentales como elemento básico de un patrimonio que debía ser común a la Unión y a los Estados. Es este ya un lenguaje privativo del TJ en cuanto conectado con la otra circunstancia que hacía especial su tarea en la materia. Por fin, también era y es singular la función complementaria de los derechos y libertades que se apunta en las palabras que cierran la cita: En la UE, los derechos fundamentales operan como premisa de cualquier adhesión a la misma de la parte de otros Estados europeos. Es esta una función que era importante subrayar cuando en el horizonte se vislumbraba la gran ampliación de la Unión que tendría lugar en $2004^{3}$.

Pero en el referido momento concurría otro factor o circunstancia bastante más peculiar de la Unión que hacía igualmente especial la ya dicha responsabilidad del TJ. Se trata de la ausencia en el ordenamiento comunitario, y a diferencia del caso de los Estados miembros, de un "catálogo», así lo califica Rodríguez Iglesias, de estos derechos fundamentales. Tengo la impresión de que el concreto término elegido, el de "catálogo», apuntaba a la idea de un mero enunciado, un listado de derechos, acaso desprovisto de la fuerza ordenadora que luego tendrá la CDF. Lo esencial a retener es que la referencia al rango - legal o constitucional- del eventual catálogo aludía directamente a su condición de derecho escrito, obra del legislador de la Unión. En seguida se volverá a la cuestión del rango. Lo que importa

3 Criterios de adhesión, Consejo Europeo de Copenhague, 1993. 
de esta otra circunstancia es su consecuencia, la que en términos abstractos cabe formular como el déficit de positividad de los derechos fundamentales tal como este se daba en el ordenamiento comunitario a la altura de la referida fecha.

Es este déficit de positividad el que habría estado durante décadas en el origen de la especial y particular responsabilidad del TJ en materia de derechos fundamentales. El ordenamiento de la Unión carecía entonces de una norma que enunciara, sistematizara y, en último término, ordenase el modo de regir de los derechos fundamentales en la UE. Rodríguez Iglesias hacía esta constatación en un momento en el que era inminente, aunque en ese momento no era discernible, el arranque en la ciudad de Tampere del proceso de diez años de duración que acabará definitivamente con el indicado déficit. Ahora bien, mientras ese momento no llegase, será al TJ a quien incumba la especial responsabilidad de subvenir a la referida carencia del derecho de la Unión. Apenas es necesario señalar que esta responsabilidad es privativa del TJ, por contraste con la de sus equivalentes en los Estados miembros que, salvo alguna excepción cualificada, se regían por constituciones escritas, catálogo de derechos incluido.

El TJ acudirá a cubrir esa laguna, obvio es decirlo, del único modo como podía hacerlo. Lo hará como juez. Lo cual supone, dicho muy elementalmente, que lo hará caso por caso y punto por punto en un proceso abierto, de larga duración, necesariamente sin sistema. Lo cual no excluía el que por la misma vía jurisdiccional quedase plasmado lapidariamente el fundamento de toda esa actividad en términos susceptibles de ser sucesivamente asumidos por el Tratado de la Unión Europea desde su primer momento: es la triada integrada por las tres categorías de principios generales, tradiciones constitucionales comunes y Convenio Europeo de Derechos Humanos (en adelante, $\mathrm{CEDH})^{4}$. Es este modelo el que cabe calificar como el del paradigma judicial como modo de existir de los derechos y libertades, vigente durante décadas, en la UE.

Pero la responsabilidad así asumida era más que todo eso, aunque Rodríguez Iglesias así expresamente no lo dijera. Porque se trata de una responsabilidad que con toda justicia ha de calificarse de histórica. Dicho muy sencillamente, en ausencia del referido «catálogo» al TJ le cupo la responsabilidad histórica de

4 Tratado de Maastricht, art. F, 2. TUE: «La Unión respetará los derechos fundamentales tal y como se garantizan en el Convenio Europeo para la Protección de los Derechos Humanos y de las Libertades Fundamentales firmado en Roma el 4 de noviembre de 1950, y tal y como resultan de las tradiciones constitucionales comunes a los Estados miembros como principios generales del Derecho comunitario». 
atraer al hoy derecho de la Unión el patrimonio común de iusfundamentalidad presente en el orden constitucional de los Estados que integran la hoy UE.

A partir de esta consideración, sería llegado aquí el momento de rendir homenaje a esta operación jurisprudencial de largo alcance que hizo posible la vigencia de los derechos y libertades en la Unión en ausencia de un catálogo de los mismos. Si la que se llamará Convención de Bruselas I pudo avanzar con pie firme en la primera versión de la CDF, ello fue posible, sin duda, gracias a la precedente tarea llevada a cabo por el TJ a lo largo de las últimas tres décadas del pasado siglo en un proceso continuo de reconocimiento y de garantía de los derechos fundamentales.

La historia es perfectamente conocida, y no es cuestión de repetir los hitos que la jalonan, desde Stauder a Internationale Handelsgesellschaft, Nold y Hauer ${ }^{5}$, y así hasta Kadi ${ }^{6}$, en tanto que momento culminante este último de la jurisprudencia de derechos fundamentales anterior a Lisboa. Solo añadiré, a efectos de completar el contexto de la cita que me sirve de hilo conductor, que Rodríguez Iglesias llega como juez a Luxemburgo en un momento coincidente con la adopción por el Tribunal Constitucional Federal alemán de la jurisprudencia conocida como Solange $I P$. No es cuestión de llevar excesivamente lejos, y menos aún en el actual momento de shock creado por la reciente sentencia de su Sala segunda ${ }^{8}$, la relevancia de la tarea reconocida al tribunal de Karlsruhe en la puesta en pie del complejo edificio jurisdiccional europeo. Con todo, cabe proponer la referida jurisprudencia como simbólica del momento en el que, desde los Estados miembros, se reconoce que la labor del TJ en materia de derechos ha adquirido la suficiente madurez como para dejar en suspenso el mandato de los tribunales constitucionales y equivalentes de asumir el control de iusfundamentalidad en cuanto extendido a los actos de los poderes públicos estatales determinados en su contenido por el hoy derecho de la Unión. Del lado de los Estados se acepta que el juicio del TJ sobre el respeto de los derechos fundamentales por los poderes públicos de la Unión pueda ser extensivo a los referidos actos

5 Sentencias de 12 de noviembre de 1969, Stauder, C-29/69, EU:C:1969:57; de 17 de diciembre de 1970, Internationale Handelsgesellschaft, C-11/70, EU:C:1970:114; de 14 de mayo de 1974, Nold, C-4/73, EU:C:1974:51; y de 13 de diciembre de 1979, Hauer, C-44/79, EU:C:1979:290.

6 Sentencia de 3 de septiembre de 2008, Kadi y Al Barakaat International Foundation/ Consejo, (Kadi I), C-402/05 P y C-415/05 P, EU:C:2008:461.

$7 \quad B V e r f G E, 73$, p. 339 ss. Sobre esta sentencia véanse Rodríguez Iglesias y Volkert (1987: 667 y ss.) y López Castillo (1988: 207-230).

8 En mi personal valoración, Cruz Villalón (2020: 1). Sentencia del Tribunal Constitucional alemán de 5 de mayo de 2020, DE:BVerfG:2020:rs20200505.2bvr085915. 
de los poderes públicos estatales, resultando así suficiente también para los tribunales nacionales. Es lo que podemos llamar el momento Solange II.

Conocido es cómo este momento supone el contrapunto y a la vez el abandono de una previa jurisprudencia nacional, por referencia siempre simbólica, de doce años atrás, reacia al abandono de su referida competencia de control jurisdiccional, y ello precisamente, conviene subrayarlo, como resultado de la constatación de una falta de presencia expresa de los derechos fundamentales en el ordenamiento jurídico comunitario9. Ha sido el largo momento Solange I. La importancia a nuestros efectos del cambio de doctrina radica en que esta se produce «a pesar de» la persistente ausencia en la UE de un catálogo de derechos y libertades puesta en evidencia en 1974. Para un nuevo Estado miembro, como era el caso de España en 1986, esto implicaba la oportunidad de sumarse directamente a este momento jurisdiccional, sin necesidad de pasar por las vacilaciones de sus socios más veteranos.

Solange II, tomada de nuevo en su carácter simbólico de un determinado momento jurisdiccional, en la medida en que traía causa de la constatación de una situación de hecho, trasladaba una especial responsabilidad al juez de la Unión en materia de garantía jurisdiccional de los derechos y libertades. La tarea histórica del TJ de atracción de los derechos fundamentales al ordenamiento de la UE era ciertamente más antigua en el tiempo, anterior incluso, como se ha visto, al momento Solange I. Pero no es aventurado considerar que esta jurisprudencia nacional operaría desde 1986 como un acicate añadido en la línea años atrás emprendida. Con ella, en suma, se abre un largo periodo en el que el TJ debe confirmar que es digno de la confianza de unos tribunales nacionales que siempre han encontrado en la garantía de los derechos fundamentales una de las marcas básicas de su identidad.

Hay que decir que los términos de esta especial responsabilidad del TJ no se alterarán con la elaboración por parte de un gremio erigido ad hoc, autocalificado de Convención, del precedente inmediato de la actual CDF, ya con su propio nombre, objeto de un simple "proclamación solemne» el 7 de diciembre de 2000 en la ciudad de Niza. Y ello sencillamente porque aquella Carta, con su eficacia jurídica dejada en tanto que tal imprejuzgada, o más bien en suspenso, no estaba en condiciones de colmar el reiterado déficit. El TJ se pasa así años haciendo de menos a aquella Carta, ignorando las invocaciones de la misma procedentes de los abogados generales, siendo solo a partir de diciembre de 2007, cuando en Lisboa ya ha quedado decidida la equiparación de aquella al derecho primario, cuando las cosas empiezan a cambiar significativamente.

9 Decisión de 29 de mayo de 1974, BVerfGE 37, p. 271 y ss. (Solange 1). 
Hay que esperar, pues, al 1 de diciembre de 2009, cuando con la entrada en vigor del Tratado de Lisboa lo haga también indirectamente la CDF, para ver el fin del reiterado déficit de positividad en materia de derechos fundamentales, tal como fuera diagnosticado ya en 1974 de la parte de los Estados miembros. Con ello desaparecerá también el fundamento coyuntural de la especial responsabilidad del TJ en lo relativo a la garantía de los derechos fundamentales. Solo que, con ello mismo también, se dará ocasión a una nueva, por diferente, manifestación de la especial responsabilidad del TJ en esta misma materia. A ello van dirigidas las páginas que restan.

\section{LA «ESPECIAL RESPONSABILIDAD» DEL TJ EN LA IMPLEMENTACIÓN DEL PARADIGMA CONSTITUCIONAL INCORPORADO POR LA CDF}

\section{EL VALOR JURÍDICO DE LA CARTA}

Es, en efecto, un precepto enormemente simple en su formulación, inserto en el Tratado de Lisboa, el que dará un giro en el sentido de la responsabilidad iusfundamental del TJ. Su tenor es de sobra conocido: «La Unión reconoce los derechos, libertades y principios enunciados en la Carta de los Derechos Fundamentales de la Unión Europea de 7 de diciembre de 2000, tal como fue adaptada el 12 de diciembre de 2007 en Estrasburgo, la cual tendrá el mismo valor jurídico que los Tratados». No hace falta decir que este precepto se corresponde con el párr. primero del apdo. 1 del art. 6 del reformulado Tratado de la Unión Europea.

La Carta, esto es lo primero que debe recordarse, ha venido obligando sin matización alguna desde la reiterada fecha como parte del derecho primario de la Unión, y también desde entonces su aplicación judicial no es distinta de la del resto del ordenamiento comunitario: sencillamente, tiene "valor jurídico", siendo esa la proclamación esencial, por más que inmediatamente se añada que ese valor jurídico es el mismo que el de los Tratados ${ }^{10}$. Entendido como proclamación de que la Carta "vale como Derecho", es claro que su valor jurídico es el mismo que el de todo el derecho de la Unión en su conjunto, el originario y el derivado. En lo que sí se puede establecer diferencias es en lo que respecta a su respectivo rango, y a esto es a lo que propiamente alude al equiparar el valor jurídico de la Carta al de los Tratados constitutivos. En suma, lo que

10 Véase a este respecto Serena Rossi (2017: 771-798). 
el TUE quiere decir, y eso explica la versión alemana ${ }^{11}$, es que la Carta tiene el rango que corresponde al derecho primario en el ordenamiento de la Unión. La CDF deviene así canon o parámetro de control de todo el derecho de la Unión que quede extramuros de dichos Tratados constitutivos ( $y$, condicionadamente, del derecho de los Estados en toda su extensión). Es así como la aplicación judicial de la CDF resulta posibilitada por la proclamación de su valor jurídico, y al mismo tiempo reforzada por la proclamación de su rango.

A partir de aquí, y sin el menor ánimo de exhaustividad, pasaré a presentar, de manera siempre sumaria, los distintos factores que han contribuido a mantener la alta responsabilidad y el fuerte protagonismo del TJ a lo largo de la primera década transcurrida de aplicación judicial de la CDF.

\section{EL VALOR CONSTITUCIONAL DE LA CARTA}

En esta línea, conviene comenzar por la referencia a un elemento que cabe englobar en la categoría de lo político-constitucional. La CDF elevada al rango de derecho primario ha supuesto un salto, acaso no cualitativo, pero en todo caso fundamental, en la dimensión constitucional de este derecho primario. Si con el resultado de los referendos de mayo de 2005 en los Países Bajos y en Francia la idea de "una Constitución para Europa» (el que hubiera sido segundo Tratado de Roma) «salía por la puerta», con el Tratado de Lisboa esta misma idea «entraba por la ventana». Convirtiendo a la Carta en derecho positivo, y evidentemente primario, de la UE, los padres de los Tratados estaban materialmente recuperando para la Unión la categoría de Constitución en una de sus dimensiones básicas. La Carta ha atraído los derechos fundamentales al espacio y a la forma del constitucionalismo moderno y al mismo tiempo ha recuperado para la Unión la representación constitucional de la misma que se hubiera dicho fracasada en 2005. Todo esto tiene para el TJ la consecuencia, en términos político-constitucionales, de poder presentarse ante el colectivo de los tribunales constitucionales y equivalentes nacionales con una reforzada veste constitucional. En definitiva, hay en este sentido argumentos para sostener que con la CDF se ha reforzado la responsabilidad constitucional del TJ.

\section{LA CDF Y LA CUESTIÓN DE LA PERVIVENCIA DEL ANTIGUO PARADIGMA}

Podría decirse que, en línea de principio, la primera responsabilidad del TJ en el inmediato periodo post-Lisboa venía marcada por el contenido del tercero y último de los apartados del mismo art. $6 \mathrm{TUE}$, que había comenzado procla-

11 Literalmente: «La Carta y los Tratados poseen el mismo rango ("sind gleichrangig")». 
mando la positividad de la Carta. En efecto, acaso lo más llamativo de la economía de este artículo sea el mantenimiento, con algunos cambios de redacción, del paradigma judicial que recibió formulación constitucional escrita en Maastricht, vale repetir, la declaración de la vigencia de los derechos fundamentales en tanto que principios generales derivados a su vez de las tradiciones constitucionales comunes a los Estados miembros y recogidos en el CEDH. Pues cabría pensar que, una vez desaparecida la premisa, la carencia en la Unión de una propia declaración de derechos, este paradigma cedería sin reservas en favor de uno nuevo que, sin pretensión alguna de desmerecimiento del anterior, podemos llamar constitucional.

No es esto, como sabemos, lo que ocurrió, de tal modo que la CDF se introdujo en el TUE sin desplazar el anterior paradigma. A lo sumo se podría hacer observar que, con arreglo a su orden de aparición en el artículo, el viejo paradigma se situaba detrás del nuevo. Llevaría lejos entrar a considerar la explicación de este sorprendente resultado, que tiene algo de esquizofrénico. Acaso pudiera aventurarse que se quiso tranquilizar al TJ con la garantía de que podría seguir operando con el antiguo paradigma, al menos en paralelo con el nuevo ${ }^{12}$. Otra explicación pudiera ser la de que se ha querido evitar una lista cerrada de derechos fundamentales, dejando abierta la posibilidad de ampliarla por medio del mantenimiento del antiguo paradigma ${ }^{13}$.

Como quiera que sea, parece claro que este esquema estaba pidiendo una intervención cuando menos orientativa por parte del TJ, a ser posible desde los primeros momentos de aplicación de la Carta. La noción más o menos difusa, posiblemente prevalente en el seno de la jurisdicción, era la de que en la UE los derechos fundamentales existen tanto en su manifestación como derechos formulados e integrados en una declaración de derechos, la CDF, como en tanto que principios generales deducibles de las fuentes de reconocimiento sabidas ${ }^{14}$.

Siendo todo esto así, cabría sostener que el TJ tenía una cierta responsabilidad en orden a sentar doctrina por lo que hace a la correlación entre los apdos. primero y tercero del art. 6 TUE. No lo ha hecho, salvo error por mi

12 Un caso particular es el de la correlación entre el principio de efectividad y el derecho a la tutela judicial efectiva (art. $47 \mathrm{CDF}$ ). Véase al respecto Pastor-Merchante (en prensa).

13 Pocas semanas después de la entrada en vigor de la CDF, en el asunto Kücükdeveci se recuerda el principio general del derecho de la Unión de no discriminación por razón de edad en la estela de Mangold, si bien inmediatamente se invoca el art. 21.1 CDF (Sentencia de 19 de enero de 2010, Kücükdeveci, C-555/07, EU:C:2010:21C-555/07).

14 Expresivas, en este sentido, las conclusiones de la abogada general Trstenjak de 8 de septiembre de 2011, Dominguez, EU:C:2011:559. 
parte, de tal modo que hay que recurrir a alguna declaración del sucesor de Rodríguez Iglesias en la presidencia del TJ, Vassilios Skouris, para encontrar la constancia de que en esta vertiente de su jurisdicción el TJ acude primordialmente a la Carta antes que a los principios generales, como así es realmente (Skouris, 2015: 299).

Como quiera que sea, aquí hay que dar ya entrada a la peculiaridad de un sistema en el que la jurisdicción de derechos fundamentales tiene básicamente lugar a través del impulso indirecto de los jueces nacionales por la vía de la cuestión prejudicial. Han sido, en efecto, ellos los que han acudido al $\mathrm{TJ}$ en petición de interpretación del derecho de la Unión «a la luz de», no de los derechos fundamentales en tanto que derivados de principios generales, sino directamente de los enunciados de la CDF. En suma, fuese cual fuese la preferencia del TJ por un paradigma y otro, han sido las preguntas contenidas en las cuestiones prejudiciales las que pronto inclinaron la balanza en favor del nuevo paradigma.

\section{LA CDF EN EL CONTEXTO DEL MANDATO DE ADHESIÓN AL CEDH}

El art. 6 TUE contenía un último capítulo en el marco de las responsabilidades reservadas al TJ en materia de derechos fundamentales para esta primera época. Su apartado segundo recoge el mandato a las instancias políticas de la Unión, de obtener el acceso de la UE al sistema regional de garantía de los derechos y libertades que representa el CEDH. Por medio de un convenio internacional singularmente mencionado en el art. 218 del Tratado de Funcionamiento de la UE (en adelante, TFUE) ${ }^{15}$, la Unión se adheriría al Convenio de Roma. No era este un objetivo novedoso por parte de las instancias políticas de la Unión ${ }^{16}$. Pero ahora se trata ya de un mandato constitucional dirigido a estas mismas instancias políticas, con la peculiaridad de que, por efecto de dos razones combinadas, el TJ tendría reservada en este proceso una responsabilidad casi determinante. De un lado, una razón procesal: el TFUE abre con notable amplitud la posibilidad de un control jurisdiccional previo de los acuerdos internacionales, como así se dice, previstos ${ }^{17}$. De otro, una razón sustantiva: pues, mientras que en lo que se refiere al enunciado de este

15 «ii) acuerdo de adhesión de la Unión al Convenio Europeo para la protección de los derechos humanos y de las libertades públicas» [art. 218.6, a) TFUE].

16 Dictamen de 28 de marzo de 1996, 2/94, Adhesión de la Comunidad al Convenio para la protección de los derechos humanos y las libertades fundamentales, EU:C:1996:140.

17 Art. 218.11 TFUE. 
apdo. 2 no hay mucho que interpretar, cosa muy distinta sucede cuando se atiende a las cautelas que el mismo Tratado de Lisboa introduce respecto del modo de la implementación del mandato. Dicho sencillamente, la adhesión solo podrá llevarse a cabo en el respeto de las «especificidades» del derecho de la Unión ${ }^{18}$. Y a diferencia de lo que es el caso del apdo. 2, aquí se abría una labor de interpretación de altos vuelos. Es tan evidente que cláusulas de este tenor postulan el concurso del supremo intérprete del derecho de la Unión que, con su sola inserción en los tratados, y por una elemental prudencia, el TJ tenía asegurado el control previo del acceso de la UE al CEDH, lo que es tanto como decir el control de la incorporación al ordenamiento de la Unión de un sistema de revisión externa de la jurisprudencia del propio TJ en materia de derechos fundamentales. Cabe observar que este control previo de dicha adhesión tenía mucho de inédito por contraste con el modo como los Estados miembros accedieron en su día al sistema del CEDH.

No es este el momento de entrar en detalle en la doctrina sentada por el TJ en el Dictamen 2/13 que declaró los acuerdos previstos de adhesión incompatibles con los Tratados constitutivos de la UE. Sí hay, sin embargo, lugar a proponer el 18 de diciembre de 2014, fecha del dictamen, como el hito más relevante de la década, no solo para la materia de los derechos fundamentales en la Unión, sino también para la posición de la Carta en el conjunto del sistema. Situado justo en la mitad de la década, el dictamen marca a ambos efectos un antes y un después. Si durante los primeros cinco años de vida activa de la Carta pudo mantenerse la expectativa de una Unión en definitiva equiparada a los Estados miembros en lo que hace a su doble obediencia a su respectiva Constitución y al CEDH, a partir de esa fecha esa expectativa simplemente se esfuma, como los cinco años transcurridos desde entonces lo confirman.

No es necesario argumentar mucho para poner de relieve lo que la relegación sine die de la adhesión al CEDH supone para el modo de regir de los derechos y libertades en el ámbito de la UE. Más interés tiene en el actual contexto la repercusión del sentido del referido dictamen sobre el modo como la CDF se aplica. El CEDH está ciertamente presente en el sistema jurídico de la Unión de varias maneras. Una de ellas es en forma del mandato de

18 Protocolo (n. ${ }^{\circ}$ ) sobre el apartado 2 del artículo 6 del Tratado de la UE relativo a la adhesión de la Unión al CEDH, art. 1: «El acuerdo...estipulará que se preserven las características específicas de la Unión y del Derecho de la Unión, en particular...»; Declaración (n. ${ }^{\circ}$ 2) relativa al apartado 2 del artículo 6 TUE: «La Conferencia conviene en que la adhesión de la Unión...debería realizarse de manera que se preserven las especificidades del ordenamiento jurídico de la Unión...». 
adhesión que se ha visto. Pero hay otras, también relevantes, alguna ya mencionada, como es su función como fuente de reconocimiento de los derechos fundamentales en tanto que principios generales. $\mathrm{Y}$ sobre todo está la otra: el mandato de alineación de los derechos de la CDF con los del $\mathrm{CEDH}$, cada vez que se constate una identidad en lo formulación de unos y otros, siempre salvada la eventualidad de un superior nivel de protección en el derecho de la Unión (art. 52.3 EDF). Aparentemente todo esto no se ha visto afectado por el dictamen. Ocurre sin embargo que, como consecuencia del sentido de este último, la interpretación que el TJ haga de estas cláusulas, la del art. 6.3 TUE y la del art. 52.3 CDF, no podrá ser revisada por el TEDH. Y, de modo más general: la jurisprudencia del TJ en materia de derechos fundamentales es firme y definitiva, con la sola reserva de que la jurisprudencia Bosphorus del TEDH sea en algún momento reconsiderada ${ }^{19}$.

\section{EL TJ ANTE LAS INDEFINICIONES DEL TíTULO VII DE LA CARTA}

La mirada ha de desplazarse ya del art. 6 TUE al Título VII y último de la Carta. Es aquí, en efecto, donde se sitúa un amplio arsenal de nociones llamadas a centrar la atención del TJ. Bajo el expresivo epígrafe de "Disposiciones Generales que rigen la interpretación y aplicación de la Carta», los cuatro preceptos que integran este Título vienen a contener el «sistema operativo» de la misma. Si se quiere decir de otro modo, son las «instrucciones de uso» de la CDF, destinadas de modo muy particular al TJ. Podría pensarse que, a partir de estas disposiciones, al TJ le quedaba poco verdaderamente relevante que añadir en términos de acabado final del nuevo paradigma (Cartabia, 2017: 1-17). No era ese, ni de lejos, el caso. Del conjunto de estos últimos preceptos de la Carta se deriva de nuevo una «especial responsabilidad» para el TJ durante el periodo inaugural que ahora contemplamos. En el contexto de esta presentación apenas cabrá otra cosa que un simple enunciado de los principales campos abiertos al trabajo interpretativo del $\mathrm{TJ}^{20}$.

En este sentido, lo primero a notar es una circunstancia que, en línea de principio, debiera contribuir a rebajar el protagonismo del TJ en estos primeros años de vigencia de la Carta: se trata de las llamadas «explicaciones» que originariamente se hicieron con la intención de subrayar que era poco lo

19 Sentencia del Tribunal Europeo de Derechos Humanos de 30 de junio de 2005, Bosphorus Hava Yollari Turizm ve Ticaret Anonim Sirketi c. Irlanda, ECHR:2005:0630 JUD004503698.

20 Dejo conscientemente de lado clásicos de tanta entidad como la eficacia horizontal de los derechos, o el de la potencialidad federalizante de los derechos, entre otros. 
que en materia de derechos la convención redactora de la CDF innovaba ${ }^{21}$. Desde aquel momento de Niza, estas «explicaciones» han visto reforzado progresivamente su estatus, apareciendo, casi machaconamente, en el art. 6 TUE, en el preámbulo de la CDF y en su art. 52.7. Lo interesante de esas «explicaciones» es que frecuentemente no hacen sino recoger la jurisprudencia anterior del propio TJ: en este sentido tienen frecuentemente el sentido de una exhortación dirigida al TJ de no apartarse de su propia jurisprudencia. Ahora bien, una verdadera ayuda para el TJ en lo que se refiere a su tarea de subvenir a las grandes indefiniciones del Título VII no se puede decir que hayan sido.

Si hubiera que destacar un solo problema expresivo de la responsabilidad del TJ en el conjunto de este Título VII, habría acuerdo en citar la cuestión del «ámbito de aplicación» de la CDF en los Estados miembros. Ningún pasaje de la Carta suscitaba tanta expectación como el contenido en la sencilla docena de palabras con la que concluye el primer inciso de su art. 51.1: «[...] así como a los Estados miembros únicamente cuando apliquen el Derecho de la Unión». Por medio de ellas el legislador constitucional de la Unión proclamaba la aplicación directa de la Carta al ordenamiento de los Estados miembros. Para el TJ estas pocas palabras significaban la atracción a su competencia del control último de iusfundamentalidad de los correspondientes actos nacionales.

Desde el principio se ha destacado la relevancia que para el desarrollo del paradigma judicial tuvo la jurisprudencia constitucional nacional en relación con aquella parte de los ordenamientos nacionales que podía considerarse directamente determinada por el derecho de la Unión. A quién debiera corresponder la concreción de ese alcance es algo que ha sido siempre de vital importancia para los Estados. Cualquier término verbal, al igual que cualquier adverbio modal que pretendiera describirlo no disminuiría el reto de su interpretación. Y sin que, una vez más, las referidas explicaciones añadieran gran cosa.

Sea como sea, no se puede decir que en esta ocasión el TJ haya rehuido su responsabilidad en el esclarecimiento de este trascendental extremo. Apenas dos años después de la entrada en vigor de la CDF, ya sentaba jurisprudencia en un asunto cuyo cuadro fáctico y normativo no debía dar lugar a dudas sobre el alcance del entendimiento del crítico pasaje del art. 51.1 CDF: Åkerberg, el nombre del asunto, pasó desde el primer día a simbolizar una doctrina inequívocamente expansionista del alcance del

21 Explicaciones sobre la Carta de los Derechos Fundamentales (2007/C 303/02), DOUE de 14 de diciembre de 2007. 
precepto $^{22}$. Apenas es necesario decir que esta jurisprudencia no recibió el aplauso unánime de los tribunales nacionales. Al mismo tiempo hay que señalar que, prácticamente desde el principio, esta jurisprudencia fue siendo objeto de matizaciones puntuales, todo ello desde luego sin poner en cuestión el planteamiento de partida ${ }^{23}$.

Por cierto, que del mismo día es la sentencia recaída en el asunto Melloni²4. Y con ello cabe dar entrada a otro capítulo de la responsabilidad del TJ en el inmediato periodo post-Lisboa. El caso es que el art. $53 \mathrm{CDF}$, en la medida en que parecía dejar en todo caso incólumes eventuales niveles superiores de protección de los derechos que pudieran darse en los ordenamientos nacionales, podía suponer un importante reto para la unidad y coherencia del derecho de la Unión: incluso las previsiones normativas nacionales determinadas en su contenido por el derecho de la Unión deberían estar sometidos al superior canon constitucional del Estado miembro. Con esto queda dicho hasta qué punto la respuesta a dar en Åkerberg estaba conectada con el alcance a dar en el art. 53. Una concepción amplia del ámbito de protección hacía tanto más grave para la unidad y coherencia del derecho de la Unión la eventual operatividad de la cláusula del art. $53 \mathrm{CDF}$. En estos términos, una vez que se ha optado por una interpretación extensiva de la cláusula «ámbito de aplicación» se hace necesario conectarla al «nivel de protección». Es lo que se hace ya en Akerberg pero con la vista puesta en Melloni. La conclusión, en todo caso, es que en ese día 26 de febrero de 2012 quedaron por tanto despejadas por el TJ dos importantes incógnitas de la Carta, lo que hace de esta fecha el segundo hito posiblemente más relevante en la historia de estos diez años.

Otra circunstancia que contribuye aun a realzar la responsabilidad del $\mathrm{TJ}$ en este primer periodo es el escaso auxilio que puede recibir del legislador ordinario a la hora de completar el contenido sustantivo de cada derecho o libertad. Trataré de presentar este punto dando un pequeño rodeo. En el sistema operativo de la Carta ocupa una posición central el régimen de las que la Carta llama «limitaciones» de los derechos pero que más usual y expresivamente se conocen como «restricciones» en el ejercicio de los mismos. Son varias las condiciones que el art. 52.1 establece en orden a la legitimidad de estas restricciones: previstas por la ley, respeto del contenido esencial, proporcionalidad, esencialmente. En mayor o menor medida, todas ellas han requerido

22 Sentencia de 26 de febrero de 2013, Åkerberg Fransson, C-617/10, EU:C:2013:105.

23 Sentencia de 19 de noviembre de 2019, TSN y AKT, C-609/17 y C-610/17, EU:C: 2019:981.

24 Sentencia de 26 de febrero de 2013, Melloni, C-399/11, EU:C:2013:107. 
del TJ una labor de interpretación, pero la concreción del contenido esencial puede recibir atención especial en nuestro actual contexto.

A diferencia de lo que usualmente se produce en la arquitectura constitucional de los Estados miembros, en el caso de la UE no podrá hablarse de un desarrollo normativo de la Constitución, y en particular de sus derechos fundamentales. La legislación subconstitucional que en otros ordenamientos sirve para superar el laconismo con el que se enuncian los derechos fundamentales no se da en este caso y en eso la CDF se asemeja al CEDH. Tratándose del sistema del CEDH, y casi habría que decir que por la naturaleza de las cosas, los derechos solo se cargan de contenido en virtud de la jurisprudencia del TEDH. En el caso de la Unión, por el contrario, hay presente una potestad legislativa que bien hubiera podido asumir la referida función de complemento normativo. Con una puntual y muy particular excepción ${ }^{25}$, nada prevé la Carta al respecto. Cierto es que el derecho derivado deja ver la anterior presencia de un alto número de previsiones expresas que, al hilo de la regulación de las más diversas materias, hacen referencia a derechos que ahora recoge la CDF. Y a ellas se remiten ocasionalmente las "explicaciones» que la acompañan ${ }^{26}$. Pero el centro de gravedad de esta dispersa legislación rara vez se encuentra en el derecho fundamental de referencia. Este conjunto de circunstancias se traduce en una adicional fuente de responsabilidad para el TJ. Así y muy en particular, el señalado déficit de legislación de desarrollo de los derechos fundamentales se proyecta a la hora de identificar el contenido esencial de cada uno de los derechos y, consecuentemente, en la tarea de fijar el límite de las restricciones legítimas de los mismos (Lenaerts, 2019: 779-793).

Cerraré esta sencilla selección de algunos de las nociones del Título VII que reclamaban la intervención del TJ con la que muy posiblemente sea la más notable ambigüedad de la CDF. En efecto, hay que llegar al Título VII de la Carta para saber que en la misma se contienen «derechos» y se contienen "principios». Bien es verdad que el art. 6.1 TUE hablaba de "derechos», «libertades» $y$ "principios», pero los dos primeros se usan con frecuencia indistintamente y el tercero es lo suficientemente ambiguo como para pensar que es otra forma aún de designar a los anteriores. En todo caso, que hubiera dos categorías de contenidos sustantivos en la CDF cada una con su eficacia

5 Véase infra nota 29.

26 A título de ejemplos, explicación relativa al art. 11 — Libertad de expresión e información-; explicación relativa al art. 16 —-Libertad de empresa—; explicación relativa al art. 17 -Derecho de propiedad-. 
propia es, por así decir, la última de las sorpresas que la Carta reservaba. Y, con ser importante esta sorpresa, aún lo era más la indefinición en la que la Carta dejaba la identidad respectiva, de derechos o de principios, de los distintos contenidos sustantivos de la misma. La realidad es que el grado de indefinición resultante, muy posiblemente, ninguna Constitución nacional se la hubiera permitido. Por lo demás, tampoco esta vez las explicaciones que acompañan la Carta iluminan en modo apreciable el camino del intérprete. Pero las cosas no siempre son como uno se las imagina y el $\mathrm{TJ}$ ha podido sortear hasta ahora el campo de minas que este capítulo, sin ánimo de dramatizar, representa. Como en otras ocasiones, se diría que ha lanzado a los abogados generales por delante, un poco a hacer cada uno la guerra por su cuenta ${ }^{27}$.

\section{EL TJ ANTE LAS CARENCIAS DE LA TUTELA JUDICIAL DE LOS DERECHOS FUNDAMENTALES}

Lo que queda en relación con el Título VII es una carencia. Las disposiciones generales de la CDF guardan un llamativo silencio respecto de un elemento que, en línea de principio, hubiera debido constituir uno de sus principales contenidos. Se trata de la cuestión relativa a los remedios procesales, que en el constitucionalismo histórico se llamaban "garantías», es decir, los institutos que hacen operativos estos derechos para los ciudadanos mediante las herramientas procesales adecuadas (Ward, 2017: 162-185).

Sabido es cómo esta materia es central al sistema del CEDH, el cual, si ha alcanzado la relevancia conocida, ha sido precisamente gracias al instrumento de la «demanda individual». Como también es sabido cómo no son pocos los tribunales constitucionales nacionales que incorporan un específico remedio procesal a disposición de los ciudadanos para la tutela de los derechos fundamentales, en España con el nombre de recurso de amparo.

No es este desde luego el caso del ordenamiento de la Unión, y en particular, de la CDF, como tampoco del Estatuto del TJ. Acaso sea esta la razón por la cual los sucesivos presidentes del TJ, desde la entrada en vigor de la CDF, han declarado «enfáticamente» que el $\mathrm{TJ}$ «no es un tribunal de derechos humanos»: por la falta de un específico remedio procesal de tutela a la disposición de

27 Conclusiones de la abogada general Verica Trstenjak de 8 de septiembre de 2011, CIT. N. 14, EU:C:2011:559; Conclusiones del abogado general Pedro Cruz Villalón de 18 de julio de 2013, Association de Médiation Sociale, C-176/12, EU:C:2013:491; y Conclusiones del abogado general Yves Bot de 29 de mayo de 2018, Bauer y Willmeroth, C-569/16, EU:C:2018:337. 
todos. Todo ello sin entrar ahora a valorar la corrección de esa propuesta relación de causa a efecto ${ }^{28}$.

A lo largo de la CDF, en efecto, y en particular en su Título VII, resulta imposible encontrar previsión específica alguna relativa al modo como los derechos fundamentales se tutelan. Ahora bien, una matización debe en seguida hacerse, toda vez que la CDF contiene una previsión específica al respecto, pero ocurre que precisamente lo hace con el objetivo de restringir el alcance de la tutela de los "principios» en el sentido de los arts. 51.1 y 52.5 $\mathrm{CDF}^{29}$. Aparte de eso, no hay nada.

En sí misma y de modo general, esta ausencia de referencia expresa no es particularmente grave, si bien bajo la condición de que el sistema general de tutela judicial de los derechos e intereses legítimos esté configurado y funcione adecuadamente. En este aspecto, se puede decir que la CDF ha hecho su trabajo, en el sentido de que dedica todo el Título VI a la materia de "Justicia», del que forma parte el art. 47 con el expresivo epígrafe de «Derecho a la tutela judicial efectiva y a la independencia judicial». Con arreglo a su contenido, este artículo no difiere esencialmente de sus equivalentes en el CEDH y en las constituciones nacionales.

El problema, sin embargo, estriba en la particular configuración de la jurisdicción en la UE, concretamente en lo relativo a las fuertes condiciones impuestas para la viabilidad de los recursos directos, en definitiva, los recursos individuales, con el consiguiente traslado de la responsabilidad de la tutela judicial a la jurisdicción de los Estados, apoyados en la tarea por el TJ mediante la herramienta de la cuestión prejudicial. Es conocido cómo, de un lado, la jurisprudencia desde antiguo establecida y persistentemente mantenida por el TJ, léase Plaumann ${ }^{30}$, ha endurecido considerablemente estas condiciones de

28 Me he ocupado de ello en «Los derechos fundamentales y la identidad del Tribunal de Justicia», en: J. I. Ugartemendía, A. Sáiz Arnáiz y M. Morales, La jurisdicción constitucional en la tutela de los Derechos Fundamentales de la UE, Oñati, Instituto Vasco de Administración Pública, 2017, pp. 11-24.

29 Art. 52.5: «Las disposiciones [...] que contengan principios [...] sólo podrán alegarse ante un órgano jurisdiccional en lo que se refiere a la interpretación y control de la legalidad de dichos actos», es decir, los actos eventualmente adoptados por la Unión o los Estados en orden a la aplicación de aquellas.

30 Sentencia de 15 julio de 1963, Plaumann/Comisión, 25/62, EU:C:1963: «[...] que quienes no sean destinatarios de una Decisión sólo pueden alegar que ésta les afecta individualmente cuando dicha Decisión les atañe debido a ciertas cualidades que les son propias o a una situación de hecho que les caracteriza en relación con cualesquiera otras personas y, por ello, les individualiza de una manera análoga a la del destinatario; que en el presente caso, la Decisión impugnada perjudica a la demandante en cuanto 
acceso al mismo. Por otro lado, son también conocidos los límites objetivos de un mecanismo como la cuestión prejudicial para fungir como remedio individual de tutela ${ }^{31}$.

Ocurre, sin embargo, que el Tratado de Lisboa no solo ha otorgado carta de naturaleza jurídica a la CDF, sino que también ha modificado puntualmente las condiciones de acceso de los particulares al TJ mediante una prudente reformulación del precepto correspondiente del TFUE ${ }^{32}$. La nueva fórmula venía con la inequívoca intención de que no se produjesen situaciones de desamparo por el juego combinado de las limitaciones de acceso a los tribunales nacionales y al TJ.

Este es muy sucintamente expuesto el cuadro general en el que se sitúa la responsabilidad del TJ en esta específica materia de la tutela judicial de los derechos fundamentales. En perspectiva comparada, el TJ pertenece sin duda más al género de los tribunales supremos con competencia general que no al de los tribunales constitucionales, en particular al de aquellos con una singularizada competencia de tutela de los derechos y libertades. Y habría que añadir que el TJ se encuentra más que cómodo en esa condición. Dicho todo lo cual, es claro que, referido singularmente al periodo que estamos examinando, el TJ tenía una especial responsabilidad, cuando menos, en favorecer un replanteamiento de la tutela de los derechos fundamentales en la Unión.

En un intento de brevísimo balance de este capítulo cabría decir que el TJ ha combinado un mantenimiento a ultranza de su restrictiva doctrina sobre el acceso a los recursos directos combinado con un esfuerzo por extraer todas las posibilidades de tutela que ofrece la cuestión prejudicial y, muy en particular, de la variante de esta última llamada «procedimiento prejudicial de urgencia», o PPU. En definitiva, por una parte, Plaumann sigue gozando de excelente salud, sin que la modificación incorporada en el TFUE haya tenido consecuencias ni para la tutela judicial de los derechos ni para la específica

ésta es importador de clementinas, es decir, en razón de una actividad comercial que, en cualquier momento, puede ser ejercida por otro sujeto".

31 Límites derivados del efecto combinado de la necesidad de un acto de aplicación del derecho de la Unión por las autoridades nacionales unido a la discrecionalidad del juez nacional a la hora de plantear la cuestión prejudicial, discrecionalidad solo restringida en el ordenamiento constitucional de algunos Estados miembros. Véase la Sentencia de 28 de abril de 2015, T \& L Sugars y Sidul Açúcares/Comisión, C-456/13 P, EU:C:2015:284, y Conclusiones del abogado general Pedro Cruz Villalón, de 14 de octubre de 2014, T \& L Sugars y Sidul Açúcares/Comisión EU:C:2014:2283.

32 Art. 263.4: desaparición del requisito de la afectación «individual» respecto de los «actos reglamentarios que la afecten directamente y que no incluyan medidas de ejecución». 
de los derechos fundamentales. Por otra, sin embargo, la centralidad de la cuestión prejudicial en la tutela de los derechos se ha visto consiguientemente reforzada con el superior estatus adquirido por éstos desde diciembre de 2009: la jurisprudencia más relevante sobre derechos fundamentales ha tenido lugar, sin discusión alguna, por la vía de la cuestión prejudicial. En este contexto, hay que destacar cómo el TJ ha volcado sus energías sobre el procedimiento prejudicial de urgencia como el más apto sucedáneo de los procedimientos ad hoc de tutela de los derechos fundamentales. El TJ, en efecto, no ha escatimado esfuerzos materiales o personales en conseguir que, en el término aproximado de dos meses previsto, estos asuntos se vean resueltos ${ }^{33}$.

\section{CONCLUSIÓN}

El TJ no ha visto concluida su especial responsabilidad en lo que concierne a la garantía de los derechos fundamentales como consecuencia de la introducción en el ordenamiento de la UE de un paradigma constitucional en lo que hace a esta dimensión de su función jurisdiccional, es decir, con la introducción de un modelo de garantía jurisdiccional, representado por la CDF, claramente alineado con el prevalente en los ordenamientos constitucionales de los Estados miembros. Por el contrario, debido a una pluralidad de razones concurrentes, los diez años de vigencia de la CDF muestran un periodo temporal individualizable, marcado por un protagonismo del $\mathrm{TJ}$ que apenas cede en importancia respecto del que, por otros motivos, hubo de asumir en las décadas precedentes. Sería incorrecto, sin embargo, decir que una vez acotado este periodo, al TJ no le queda por delante sino un camino trillado. Por el contrario, algunas de las opciones que el TJ ha ido proponiendo en estos años le sitúan, con frecuencia de forma inmediata, ante la responsabilidad de enfrentar otros retos: la tarea de enunciarlos debe quedar ya para otra ocasión.

\section{Bibliografía}

Cartabia, M. (2017). Convergenze e divergenze nell'interpretazione delle clausole finali della Carta dei diritti fondamentali dell'Unione europea. Rivista AIC, 3, $1-17$.

33 Véanse, a título de ejemplos, los asuntos Mercredi (C-497/10 PPU), West (C-192/12 PPU), Lanigan (C-237/15 PPU). 
Cruz Villalón, P. (2017). Los derechos fundamentales y la identidad del Tribunal de Justicia. En J. I. Ugartemendía, A. Sáiz Arnáiz y M. Morales (dirs.), La jurisdicción constitucional en la tutela de los Derechos Fundamentales de la UE (pp. 11-24). Oñati: Instituto Vasco de Administración Pública.

- (2020). Exit Karlsruhe. El País, 13 de mayo.

Lenaerts, K. (2019). Limits on Limitations: the essence of Fundamental Rights in the EU. German Law Journal, 20, 779-793. Disponible en: https://doi. org/10.1017/glj.2019.62.

López Castillo, A. (1988). La cuestión del control de constitucionalidad de las normas comunitarias de Derecho derivado en la República Federal alemana. Revista Española de Derecho Constitucional, 23, 207-230.

Pastor-Merchante, F. (2020). The overlap between the principles of effectiveness and effective judicial protection in Union Law. En C. Izquierdo Sans, C. Martínez Capdevila, M. Nogueira Guastavino (eds.), Fundamental Rights Challenges Horizontal Effectiveness, Rule of Law and Margin of Appreciation. Viena: Springer Verlag (en prensa).

Rodríguez Iglesias, G. C. y Volkert, U. (1987). Derecho comunitario, derechos fundamentales y control de constitucionalidad: la decisión del Tribunal Constitucional federal alemán de 22 de octubre de1986. Revista de Instituciones Europeas, 14 (3), 667-686.

Serena Rossi, L. (2017). Same legal value as the treaties? Rank, primacy and direct effects of the EU Charter of Fundamental Rights. German Law Journal, 18, 771-798. Disponible en: https://doi.org/10.1017/S2071832200022161.

Skouris, V. (2015). Die europäische Grundrechte-Charta in der Rechtsprechung des EuGH. Arbeit und Recht. Deutsches und europäisches Arbeitsrecht, 8-9, 294-300.

Ward, A. (2017). Remedies under the EU Charter of Fundamental Right. En S. Douglas-Scott, N. Hatzis, N. (eds.). Research Handbook on EU Law and Human Rights (pp. 162-185). Cheltenham (Reino Unido): Edward Elgar Publishing. Disponible en: https://doi.org/10.4337/9781782546405.00016. 